\title{
Epidemiology of ESBL-producing Escherichia coli from repeated prevalence studies over 11 years in a long-term-care facility
}

\author{
Romain Martischang ${ }^{1 *}$ (D) Patrice François ${ }^{2}$, Abdessalam Cherkaoui ${ }^{3}$, Nadia Gaïa ${ }^{2}$, Gesuele Renzi ${ }^{3}$, \\ Americo Agostinho ${ }^{1}$, Monica Perez ${ }^{1}$, Christophe E. Graf ${ }^{4}$ and Stephan Harbarth
}

\begin{abstract}
Background: Escherichia coli sequence type (ST) 131 H30 is an emerging multidrug resistant subclone, known to spread and cause outbreaks in long-term care facilities (LTCFs).

Objectives and methods: From 2010 through 2020, we performed 11 yearly surveillance studies for determining the prevalence of digestive carriage of ESBL-producing E. coli (ESBL-EC) among residents in a university-affiliated LCTF. Sequencing and genotyping of selected isolates were performed to characterize temporal trends in the prevalence and epidemic potential of ESBL-EC subclones, and for evaluating a potential rebound effect following discontinuation of contact precautions for ESBL-EC carriers in January 2019.

Results: This study included 2'403 LTCF residents, with 252 (10.5\%) positive for ESBL-EC. Among the 236 ESBL-EC isolates available for typing, 58.0\% belonged to the ST131 lineage, including 94/137 (68.6\%) ST131 H30 isolates. An increasing yearly prevalence was observed for ESBL-EC (from 4.6 to 9.4\%; $p=0.11$ ), but not for the ST131 H30 subclone, which peaked in 2015 and declined thereafter. Multiple previously unnoticed ESBL-EC outbreaks occurred in the LTCF. Since 2018, we noted the clonal expansion of a rare ST131 H89 subclone (O16:H5) harboring CTX-M-14 and CTX-M-24. No rebound effect was observed in ESBL-EC prevalence nor in the different subclones following discontinuation of contact precautions for ESBL-EC carriers since 2019.
\end{abstract}

Conclusion: Clonal fluctuation was observed for ST131 H30 ESBL-EC with a current decline in prevalence. Surveillance should include the evolution of ST131 non-H30 subclones, which may spread in LTCFs. Our findings suggest that discontinuation of contact precautions for ESBL-EC carriers in LTCFs may be safely implemented, in support of European recommendations to limit ESBL-producing Enterobacteriaceae control measures in endemic settings to non-E. coli.

Keywords: Escherichia coli, Molecular epidemiology, PCR, ST131, Antimicrobial resistance, Fluoroquinolone resistance, Intestinal colonization, Surveillance, Long-term care facility

\footnotetext{
*Correspondence: romain.martischang@gmail.com

${ }^{1}$ Infection Control Programme and WHO Collaborating Centre on Patient

Safety, University of Geneva Hospitals and Faculty of Medicine, Geneva, Switzerland

Full list of author information is available at the end of the article
}

\section{Introduction}

The global spread of extended-spectrum beta-lactamase producing Escherichia coli (ESBL-EC) is driven by the emergence of successful clones such as E. coli ST131, particularly transmissible in long-term care facilities (LTCFs) [1, 2]. For instance, between 1996 and 2014, an increase of ESBL-EC was noticed in French LTCFs, reflecting original author(s) and the source, provide a link to the Creative Commons licence, and indicate if changes were made. The images or other third party material in this article are included in the article's Creative Commons licence, unless indicated otherwise in a credit line to the material. If material is not included in the article's Creative Commons licence and your intended use is not permitted by statutory regulation or exceeds the permitted use, you will need to obtain permission directly from the copyright holder. To view a copy of this licence, visit http://creativecommons.org/licenses/by/4.0/. The Creative Commons Public Domain Dedication waiver (http://creativeco mmons.org/publicdomain/zero/1.0/) applies to the data made available in this article, unless otherwise stated in a credit line to the data. 
clonal spread, with a $18.1 \%$ prevalence of ST131 clones $[3,4]$. In Swiss nursing homes, the proportion of ESBLEC increased from 5 to $22 \%$ between 2007 and 2017 [5].

The increasing prevalence of E. coli ST131 among LTCFs is mostly explained by the clonal expansion of emerging multi-resistant clades of ESBL-EC [6], responsible for silent clusters among residents in LTCFs [7], including the fluoroquinolone-resistant clades $\mathrm{C} 1$ (C1/ H30-R) and C2 (C2/H30-Rx) [8]. The reasons behind this apparent success remain controversial, but recent genomic and proteomic studies suggest that an improved anaerobic metabolism, as well as other human colonization and virulence factors helped this clone to outcompete the gut commensal niche [9-11], with consecutive prolonged colonization [12]. This lineage particularly fostered the community spread of CTX-M, by the maintenance of clade-restricted multi-drug resistant plasmids [13]. A nested cohort study of a large clinical trial recently observed the dominance of C1/H30-R ESBL-EC in participating European LTCFs [14]. In that study, 49\% $(16 / 33)$ of all ESBL-EC ST131 carriers in Geneva were positive for $\mathrm{C} 1 / \mathrm{H} 30-\mathrm{R}$, compared to $20-39 \%$ in the 3 other centers outside Switzerland.

Considering the excess mortality and hospital stay associated with third-generation-cephalosporin-resistant E. coli $[15,16]$, the epidemic potential of these ESBLEC clades represents an infection control challenge in LTCFs, in particular in institutions without contact precautions for ESBL-EC carriers [17]. Effectively, many LTCFs around the world have discontinued contact precautions for ESBL-EC carriers, in light of recent studies on low nosocomial ESBL-EC transmission rates and endemic community carriage [17].

In our university-affiliated LTCF, yearly prevalence surveys were conducted from 2010 to 2020 as routine surveillance strategy to monitor the epidemiology of ESBL-producing Enterobacterales (ESBL-PE). In the present study, we sought to (i) characterize the temporal trends in the prevalence of ESBL-EC subclones among LTCF residents; (ii) combine epidemiological information with sequencing approaches to estimate the epidemic potential of emergent ESBL-EC subclones; and (iii), determine a potential rebound effect after de-implementation of contact precautions for carriers of these subclones.

\section{Methods}

\section{Design and setting}

This 11-year retrospective study was constituted by yearly prevalence surveys from 2010 through 2020, performed during January-February of each year, among all LCTF residents. Eight long-term care wards from a same geographical site, representing 216 beds were included.
From 2018 onwards, we added four long-term care wards from a second site, representing 73 beds.

\section{Outcomes}

The primary outcome included the overall prevalence of ESBL-EC carriage and abundance of different subclones across years, defined as the total number of positive cases per 100 screened residents. Secondary outcomes included the overall prevalence of any ESBL-PE, the number of clusters (i.e. at least two residents sharing a ST131 H30, ST131 non-H30, and non-ST131 strain in the same ward the same year), the prevalence of subclones in the wards concerned by these clusters, and the proportion of clonally related strains among these clusters. Clonal relatedness was defined based on genomes, using a threshold in the pairwise distance of $\leq 10$ SNP differences, as suggested elsewhere [18].

\section{Infection control practices}

In addition to standard precautions, until December 2018, all identified ESBL-PE carriers were placed under contact precautions, including gloves, hydrophobic coats, and, whenever possible, isolation in single-bed rooms. Contact precautions were abandoned for ESBL-EC from January 2019 onwards, with simultaneous reinforcement of standard precautions using routine observation and feedback by infection control nurses, in particular hand hygiene.

\section{Health-related data}

Epidemiological information was prospectively collected for each participant during the surveys, including ward location, admission date, date of sampling, previous positive cultures, age, and gender. We collected yearly hand hygiene adherence of healthcare workers in LTCFs from 2014 to 2020 according to WHO methods, as well as the length of stay of all residents in the concerned wards during January and February, from 2010 to 2020.

\section{Microbiological methods}

Rectal swabs (E-swab, Copan) or stool cultures were collected for all participants, and processed using selective chromogenic agar (ChromID ESBL; bioMérieux). All colonies that met the expected chromogenic features provided in the manufacturers' specifications were identified by matrix-assisted laser desorption ionization-time of flight (MALDI-TOF) mass spectrometry (Bruker Daltonics, Bremen, Germany) and the antibiotic susceptibility profiles of each isolate was determined by the disk diffusion method using EUCAST breakpoints and recommendations [19]. Double-disk synergy tests (DDST20 and DDST30) were used for ESBL confirmation, ensuring a high sensitivity and specificity for ESBL-PE detection 
[20]. Assessment by ESBL+AmpC Screen Kit 98008 (Rosco Diagnostica, Denmark) was also performed to identify the partially derepressed AmpC whenever the results of the DDST20 and cefoxitin tests were not conclusive.

\section{Molecular typing}

Allelic discrimination qPCR assays were performed on all newly detected ESBL-EC to ascertain ST131 lineages and H30 subtypes. For known carriers, we only retained the first ESBL-EC strain if isolated in the prior 12 months. Five single nucleotide polymorphism assays targeting specific positions in 2 genes used for MLST and constituting a unique signature of ST131 were selected and validated against a collection of $>90$ sequenced strains from highly diverse genetic backgrounds, as previously described [21]. The 6th assay was created from an existing in silico PCR and targets H30 through a coding point mutation in FimH sequence. This allelic discrimination TaqMan ${ }^{\mathrm{TM}}$ system has been validated on a collection of approximately 100 clinical strains isolated from bloodstream infections in 2015 in our institution (not published). Subclades ST131 H30 were then defined according to fluoroquinolone resistance $(\mathrm{C} 1 / \mathrm{H} 30-\mathrm{R})$ and additional presence of the bla gene CTX-M-15 (C2/ H30-Rx).

\section{Sequencing and assembly}

Candidate strains for sequencing included ST131 H30 strains observed in large clusters since 2010 (with at least 4 positive cases from the same ward), and in all clusters since 2018 (with at least 2 positive ward mates). Moreover, all non-ST131 and ST131 non-H30 isolates from 2018 onwards were sequenced. Only the first isolate per patient and one morphotype per plate were considered for typing and sequencing. Purified genomic DNA (DNeasy, Qiagen) of selected isolates was sequenced using Illumina HiSeq2500 device using 100 base pairs (bp) paired-end reads and bar codes strategy according to the Nextera XT kit (Illumina), following the manufacturer's recommendations. Read quality was assessed with the Fastqc program (http://www.bioinformatics. babraham.ac.uk/projects/fastqc/) and filtered using the FastqMcf program (Ea-utils; http://code.google.com/p/ ea-utils). Genome assembly was performed using Spades assembler v 3.12.0. Assembled genomes were submitted individually to the Center for Genomic Epidemiology (https://cge.cbs.dtu.dk/) for confirmation of serotypes by using FimTyper 1.0 and SerotypeFinder 2.0.

\section{Core genome multi-locus sequence typing target genes}

The task template "E. coli cgMLST v1.0" was used in a multi-locus sequence typing (cgMLST) scheme with
Ridom SeqSphere + software version 5 (Ridom GmbH, Germany) using default settings. The final cgMLST scheme consisted of 2'513 genes covering roughly $45 \%$ of the genomic sequence of $E$. coli. From each isolate, the complete sequence of each gene was analyzed according to the cgMLST scheme and a numerical allele type was assigned to that given locus. The allelic profile was therefore determined by combining alleles of all cgMLST loci for each strain. A minimum spanning tree (MST) was inferred by neighbor joining method on the allelic profiles. The remaining genes were used for pairwise-comparisons.

\section{Statistical methods}

Proportions were compared using $X^{2}$ tests, or two-tailed Fisher's exact test when appropriate. The prevalence curve was segmented based on seeming inflection points for statistical comparison, as defined elsewhere [22]. A chi-square test for linear trend across these segments assessed prevalence shifts over time [23]. Genetic diversity was estimated using the number of ST divided by the number of strains sequenced. All analyses were conducted using R.4.0, including the package "Ime4".

\section{Results}

From January 2010 through February 2020, 11 yearly cross-sectional surveys of ESBL-PE carriage included 2'403 LTCF residents, with a median age of 83 years (IQR 75-89), and $61.4 \%$ of women. Yearly hand hygiene adherence improved from 72 to $77 \%$ from 2016 to 2020 (Additional file 1: Figure S1). The median length of stay of patients hospitalized in January and February from 2010 to 2020 decreased from 138.0 days (IQR 60.9-321.0) to 33.8 days (18.0-74.4.0). The total prevalence of any ESBL-PE carriage was $13.3 \%(\mathrm{n}=319)$ and doubled from $7.1 \%$ to $13.8 \%$ over 10 years $(p=0.04)$. Among ESBLPE positive patients, $79.0 \%(\mathrm{n}=252)$ and $18.8 \%(\mathrm{n}=60)$ were respectively colonized with E. coli and Klebsiella pneumoniae. Over the study period, ESBL-EC prevalence increased from $4.6 \%$ to $9.4 \%(p=0.11)$, with a peak of $14.2 \%$ in 2018 (Fig. 1). We observed an increase of prevalent (previously known) cases from $11.1 \%$ to $43.0 \%$ of ESBL-EC from 2010 to 2020, with a stable proportion of incident (newly identified) cases. Of note, this increase was partly driven by nosocomial clusters throughout multiple wards in 2012, 2013, 2018, and 2019 (Fig. 2).

Overall, 58.0\% (137/236) of typed ESBL-EC isolates belonged to the ST131 lineage, with $68.6 \%(94 / 137)$ positive for ST131 H30. The prevalence of this subclone remained stable until 2015 (Fig. 3A), with a subsequent downward slope deflection from 2015 to 2020 (76.5\% to $33.3 \%, p<0.003)$. No rebound effect was recorded neither for ESBL-EC, nor specifically for ESBL-EC ST131 


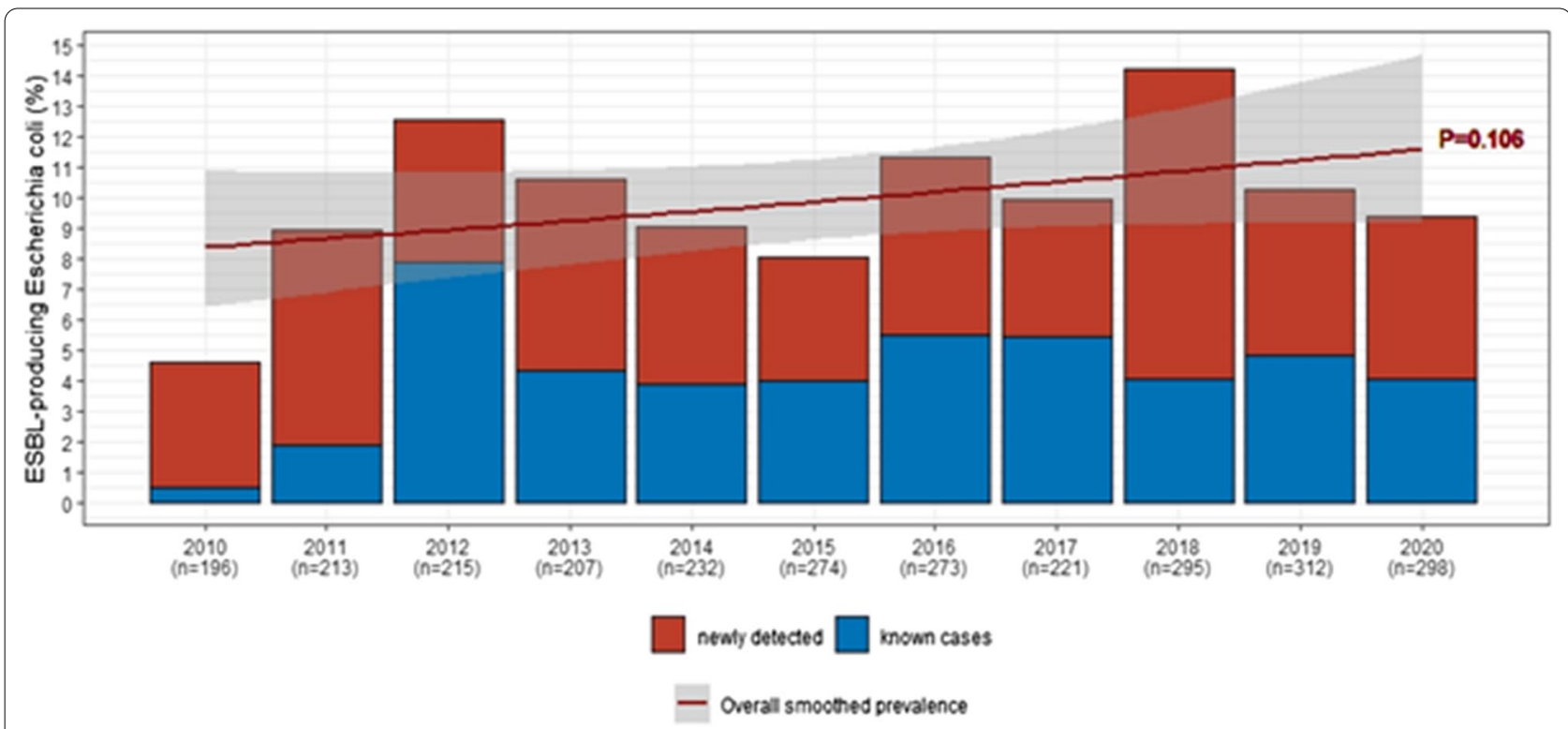

Fig. 1 Prevalence of ESBL-producing Escherichia coli carriage among all residents of a university-affiliated long-term care facility from 2010 to 2020, before and after de-implementation of contact precautions in January 2019, and stratified between previously known and newly detected carriers

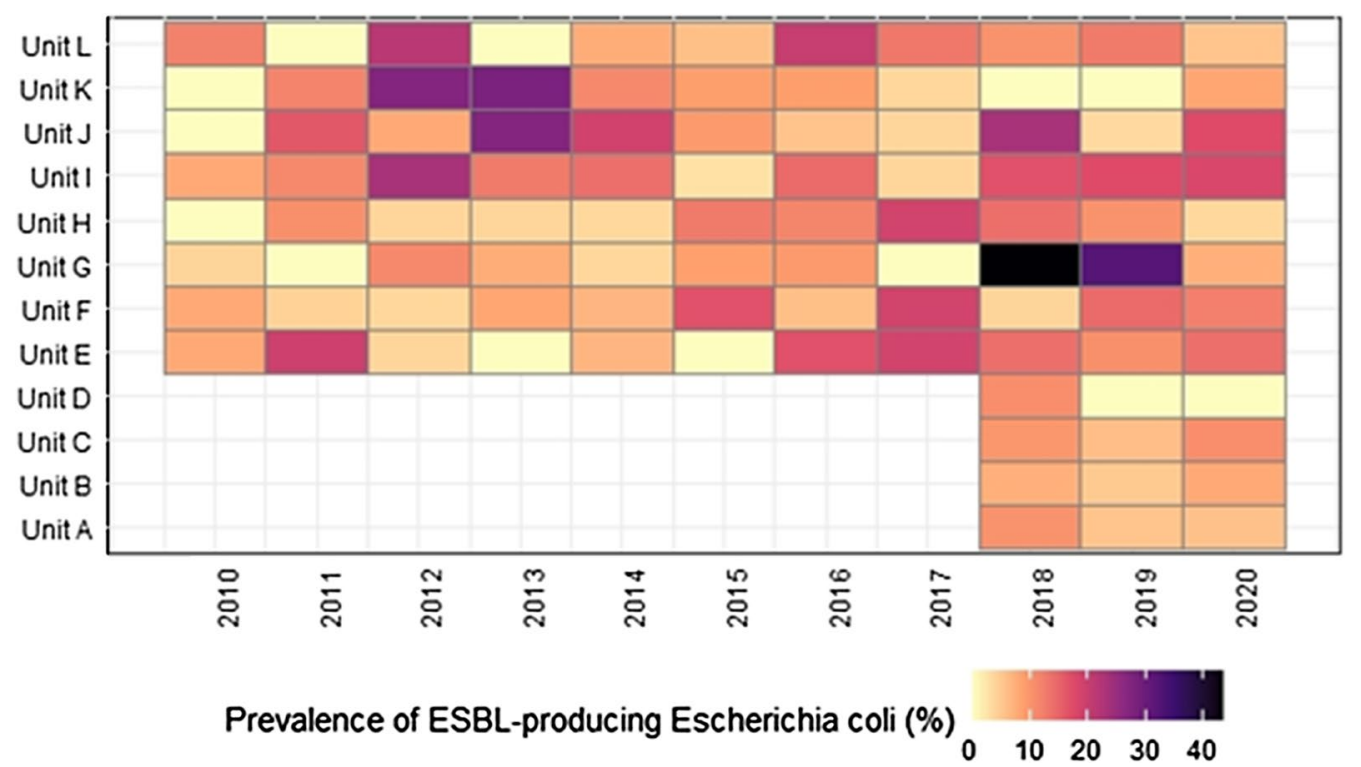

Fig. 2 Yearly prevalence and clustering of ESBL-producing Escherichia coli carriers within 12 wards of a university-affiliated long-term care facility, Geneva (2010 to 2020). From 2018 onwards, 4 long-term care wards were added from a separate facility

H30 following de-implementation of contact precautions for ESBL-EC carriers in January 2019. In contrast, we observed an increase of ST131 non-H30 subtypes from 2016 to $2020(p=0.016)$, which peaked in 2018 (Fig. 3A). In total, 82 of 236 (34.7\%) typed ESBL-EC were sequenced, including 11 ST131 H30 strains from large nosocomial clusters in 2010-2017, 10 ST131 H30 strains from clusters in 2018-2020, as well as 24 ST131 non-H30 and 37 non-ST131 strains, isolated since 2018 (Additional file 1: Table S1). Among ST131 H30 isolates, 12 belonged to the clade $\mathrm{C} 2 / \mathrm{H} 30-\mathrm{Rx}(57.1 \%)$, and 8 to the clade $\mathrm{C} 1 /$ H30-R (38.1\%; Fig. 3B). Of note, whereas the majority of $\mathrm{C} 2 / \mathrm{H} 30-\mathrm{Rx}$ strains (58.3\%) were detected in 2015, C1/ H30-R strains were only detected from 2017 onwards. 


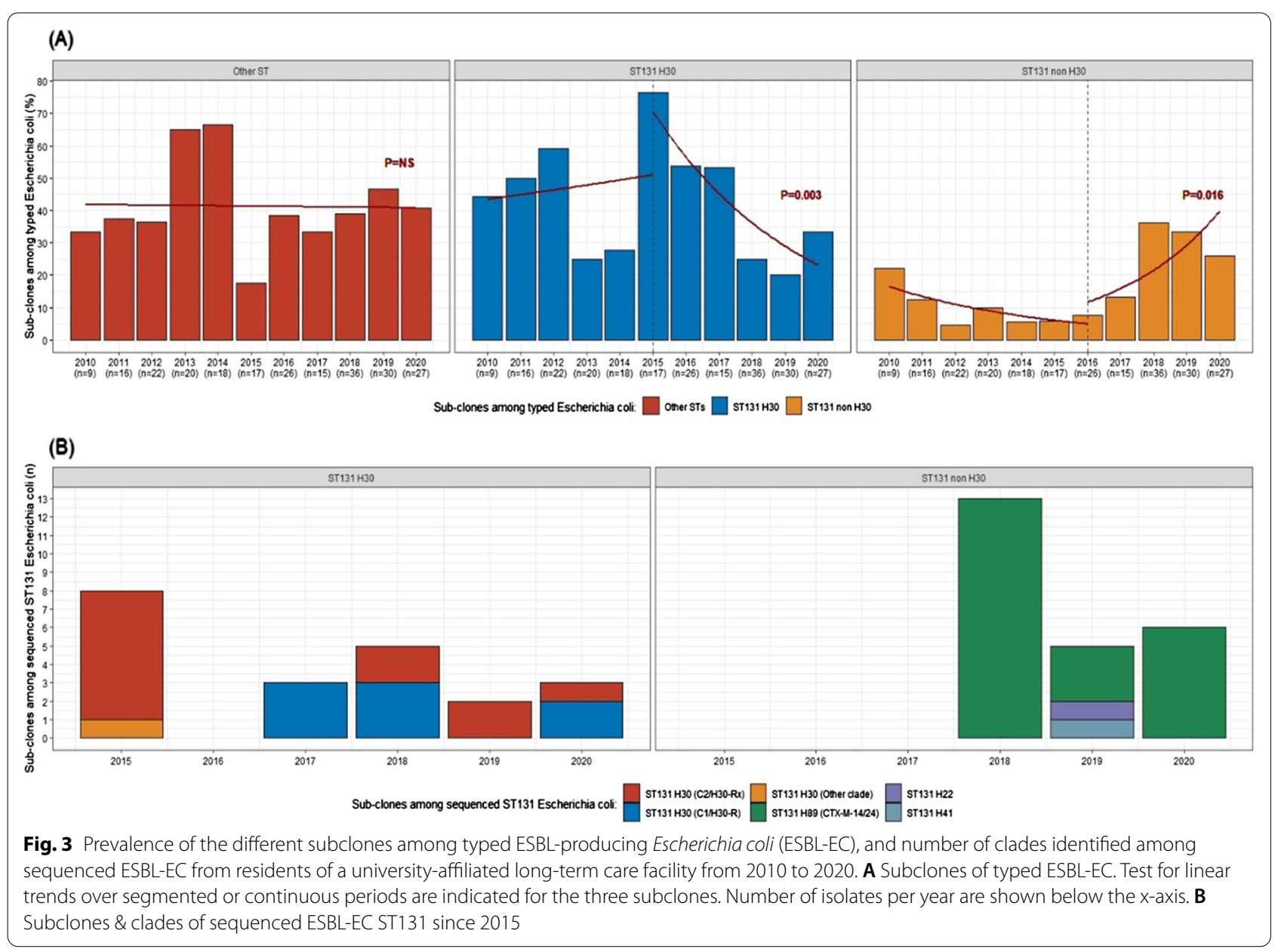

Among ST131 non-H30, we observed the emergence and expansion of $22(91.7 \%)$ isolates belonging to the ST131 H89 strain (O16:H5) associated with both CTXM-14 and CTX-M-24. Among the 37 non-ST131 isolates, 31 different sequence types were identified, precluding possible monoclonal spread. Among ST131 H30, the most common resistance genes were CTXM-15 (72.2\%), OXA-1 (55.6\%), and CTXM-27 (22.2\%). Among ST131 non $\mathrm{H} 30$, the most common resistance genes were CTXM-14 (91.7\%), CTXM-24 (91.7\%), and TEM-1B (75.0\%). Among non-ST131, the most common resistance genes were CTXM-15 (43.2\%), TEM-1B (32.4\%), OXA-1 (13.5\%), and CTXM-14 (13.5\%).

When considering epidemiological information from 2010 to 2020, we observed 27 nosocomial clusters of patients positive for ESBL-EC ST131 H30. Almost all (20/21) ESBL-EC ST131 H30 strains available for sequencing were genotypically related (Fig. 4). C2/ H30-Rx strains dominated in 2015, while C1/H30-R was present in more recent clusters. Sixteen of these 21 (76\%) strains were isolated from 2 wards (unit F and $\mathrm{H}$ ) between 2015 and 2020. Twenty of 24 (83\%) ST131 nonH30 strains available for sequencing were genotypically related and identified as ST131 H89 with the serotype O16:H5, which expressed the same CTX-M-14 and CTXM-24 genes. We observed 5 clusters of patients positive for ST131 H89 in three wards (wards G, H, and I), with an attack rate of $12 \%$ (17 of 139 susceptible patients, Fig. 3). Finally, only $18 \%$ (5/27) of sequenced non-ST131 strains were genotypically related (Fig. 4).

\section{Discussion}

The findings of these 11 yearly cross-sectional surveys support five main conclusions: (1) ESBL-EC prevalence increased over time in this university-affiliated LTCF, mainly driven by an increased proportion of previously known carriers; (2) after 2015, a decreasing prevalence of ST131 H30 subclones was observed over time, despite small localized outbreaks; (3) clonal expansion of ST131 H89 (O16:H5) subclones occurred since 2018, driven by multiple silent outbreaks; (4) We did not document the replacement by an emerging non-ST131 clone; and (5) 


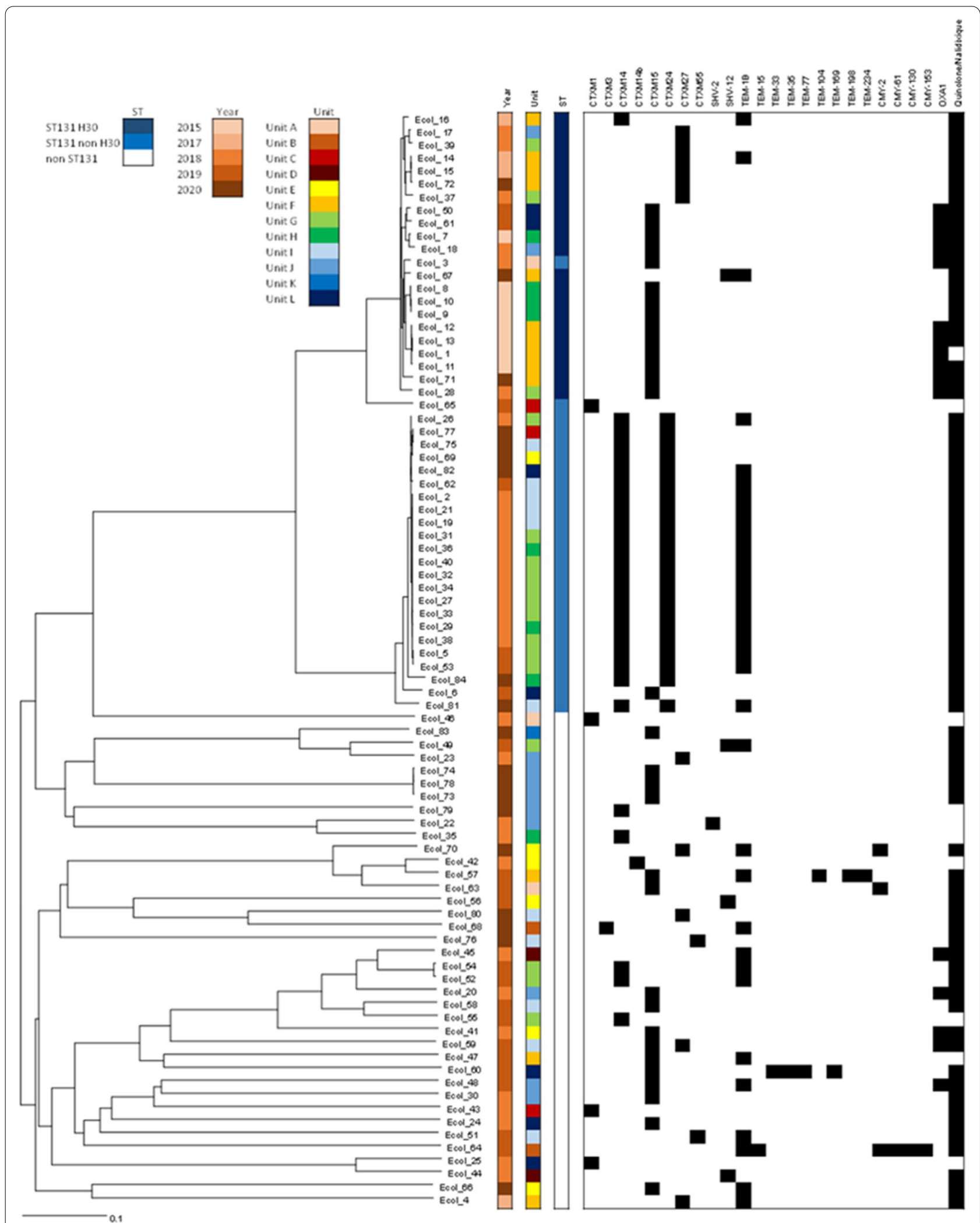

Fig. 4 Dendrogram of sequenced ESBL-producing Escherichia coli, with epidemiological information and molecular data on ESBL genes; PCRH30 represent the results from the multi-array PCR, with non-ST131, ST131 H30, and ST131 non-H30 
no rebound effect in ESBL-EC or specific subclones was observed following discontinuation of contact precautions, though longer follow-up periods are needed to validate this finding.

LTCFs are well-known reservoirs for multiresistant ESBL-EC clones, with specific patient- and care-related exposures facilitating the spread of certain clades, including vulnerable and dependent patients with prolonged lengths of stay [24-29], as well as recognized challenges in implementing infection control measures [24, 28]. Many outbreaks report silent transmission of ESBL-EC ST131 in LTCF, especially belonging to the clade C2 (C2/ H30-Rx-CTX-M-15) [1, 2, 6]. The rapid clonal expansion of this $\mathrm{C} 2$ clade through nosocomial outbreaks in LTCFs has already been observed to displace preexisting E.coli clades [6]. Thus, clonal fluctuance has been a recognized phenomenon with emergence and decline of temporarily successful clones. The persistence of certain E. coli clones, sporadically carrying carbapenemases genes, warrants further careful surveillance. [30]

Until now, few studies have reported nosocomial outbreaks associated with E.coli ST131 non-H30 clades. Population genomics on $4^{\prime} 071$ global sources genomes observed a dominance of the clade $\mathrm{C}$, co-circulating worldwide at stable frequencies [31]. In 2018, a single Spanish LTCF of 300 residents observed only 6 ST131 non-H30 associated with CTX-M-14 on 55 typed ESBLEC isolates [32]. Our study observed that neither E. coli $\mathrm{C} 2 / \mathrm{H} 30-\mathrm{Rx}$, nor $\mathrm{C} 1 / \mathrm{H} 30-\mathrm{R}$, seem to drive the recent changing epidemiology of ESBL-EC in our LTCF, but rather a ST131 H89 harboring CTX-M-14 and CTX-M27. This strain was sub-typed based on its fimH typing region, which is closely related and often associated to the H41 group of E. coli ST131 (1 SNP difference) [33$35]$. To the best of our knowledge, there has been no outbreak report of this ST131 H89 E. coli subclone, which is anecdotally reported in population genomics $[31,35,36]$.

The findings of this study are also in line with currently available evidence, which supports the discontinuation of contact precautions for ESBL-EC carriers, as suggested by European recommendations to limit ESBL-PE control measures in endemic settings to non-E. coli ESBL-PE [17]. Clonal outbreaks already occurred before discontinuation of contact precautions, and were not catalyzed by this decision. The observed recent clusters might not be an effect from lack of strict contact precautions, but rather direct consequences of the LTCF infrastructure, among other factors related to this specific setting [28, 37].

Though this study includes a large sample size and long-term surveillance data, we acknowledge the presence of several limitations. First, we could not quantify transmission and acquisition events due to the study design. Second, we acknowledge a potential bias in the selection of $E$. coli strains sequenced. Third, generalizability of our findings is impacted by the unicentric approach. Fourth, potential lack of genomic discrimination between highly similar $E$. coli clades was not possible due to the sequencing methods used (short reads). Fifth, the decreased length of stay could have impacted ESBL-EC prevalence by lowering the probability of nosocomial ESBL-EC acquisition and the proportion of known carriers. However, the acquisition risk in relation to the length of stay has been observed to be similar between ST131 and non-ST131 E. coli, and did not differ between 6 and 8 months of stay in LTCF. [12] Furthermore, ESBL-EC prevalence appears similar between LTCFs and among elderly living in the community. [38] For these reasons, the decreasing length of stay probably did not substantially influence ESBL-EC prevalence.

\section{Conclusion}

The changing ESBL-EC epidemiology, emergence of novel clones, and related clusters in LTCF, though not impacted by discontinuation of contact precautions, should be monitored by a comprehensive screening and surveillance strategy.

\section{Supplementary Information}

The online version contains supplementary material available at https://doi. org/10.1186/s13756-021-01013-7.

Additional file 1. Supplementary online content.

\section{Acknowledgements}

We thank the bacteriology laboratory for the additional workload generated yearly from 2010 for this repeated prevalence survey, Delphine Perréard for collecting data during the early study period, the genomic research lab for the additional tests performed in the context of this study, and also all LTCF residents subject to this infection control surveillance. We also thank Eve-Julie Bonetti who performed the PCR typing of all ESBL-producing E.coli included in this study.

\section{Prior presentation}

R. Martischang et al. International Conference on Prevention and Infection Control. Epidemiology of ESBL-producing Escherichia coli from repeated prevalence studies over 11 years in a long-term care facility. 2021;(O05).

\section{Authors' contributions}

$\mathrm{RM}, \mathrm{PF}, \mathrm{SH}$ were involved in study design and implementation. AC, NG, GR contributed to methodological advice. RM, PF, SH led project implementation and data collection. MP contributed to data collection. More specifically in the laboratory work, PF, GR, AC supervised the results, NG performed sequencing, assembly, and cgMLST. RM, NG contributed to data analysis and generated tables and figures. RM, SH led the manuscript writing. RM, PF, AC, NG, GR, CG, $\mathrm{SH}$ were involved in data interpretation, reviewed drafts of the manuscript and provided critical input. All authors approved the final version of the manuscript. 


\section{Funding}

The first author was partially supported by the Swiss National Science Foundation (Grant Agreement 407240_177454).

\section{Availability of data and materials}

The datasets used and/or analyzed during the current study are available from the corresponding author on reasonable request.

\section{Declarations}

\section{Ethical approval and consent to participate}

Data used in this study were collected retrospectively from our routine surveillance, therefore informed consent was not required according to the Swiss law for research on human beings.

\section{Consent for publication}

Not applicable.

\section{Competing interests}

The authors declare that they have no competing interests.

\section{Author details}

${ }^{1}$ Infection Control Programme and WHO Collaborating Centre on Patient Safety, University of Geneva Hospitals and Faculty of Medicine, Geneva, Switzerland. ${ }^{2}$ Genomic Research Laboratory, Geneva University Hospitals, Geneva, Switzerland. ${ }^{3}$ Bacteriology Laboratory, Geneva University Hospitals, Geneva, Switzerland. ${ }^{4}$ Department of Rehabilitation and Geriatrics, Geneva University Hospitals, Geneva, Switzerland.

Received: 20 July 2021 Accepted: 25 September 2021

Published online: 19 October 2021

\section{References}

1. Brodrick HJ, Raven KE, Kallonen T, Jamrozy D, Blane B, Brown NM, et al. Longitudinal genomic surveillance of multidrug-resistant Escherichia coli carriage in a long-term care facility in the United Kingdom. Genome Med. 2017;9(1):70.

2. Giufrè M, Ricchizzi E, Accogli M, Barbanti F, Monaco M, de Araujo FP, et al. Colonization by multidrug-resistant organisms in long-term care facilities in Italy: a point-prevalence study. Clin Microbiol Infect. 2017;23(12):961-7.

3. Nicolas-Chanoine M-H, Jarlier $\mathrm{V}$, et al. Extended-spectrum beta-lactamases in long-term-care facilities. Clin Microbiol Infect. 2008;14(Suppl 1):111-6.

4. Sauget $M$, Cholley $P$, Vannier $A$, Thouverez M, Nicolas-Chanoine $M-H$, Hocquet $D$, et al. Trends of extended-spectrum $\beta$-lactamase-producing Escherichia coli sequence type 131 and its H30 subclone in a French hospital over a 15-year period. Int J Antimicrob Agents. 2016;48(6):744-7.

5. Kohler P, Fulchini R, Albrich WC, Egli A, Balmelli C, Harbarth S, et al. Antibiotic resistance in Swiss nursing homes: analysis of National Surveillance Data over an 11-year period between 2007 and 2017. Antimicrob Resist Infect Control. 2018;7(1):88.

6. Ludden C, Decano AG, Jamrozy D, Pickard D, Morris D, Parkhill J, et al. Genomic surveillance of Escherichia coli ST131 identifies local expansion and serial replacement of subclones. Microb Genom. 2020;6(4):e000352.

7. Willemsen I, Nelson J, Hendriks Y, Mulders A, Verhoeff S, Mulder P, et al. Extensive dissemination of extended spectrum $\beta$-lactamase-producing Enterobacteriaceae in a Dutch Nursing Home. Infect Control Hosp Epidemiol. 2015;36(4):394-400.

8. Price LB, Johnson JR, Aziz M, Clabots C, Johnston B, Tchesnokova V, et al. The epidemic of extended-spectrum- $\beta$-lactamase-producing Escherichia coli ST131 is driven by a single highly pathogenic subclone, H30-Rx. MBio. 2013;4(6):e00377-13.

9. Kallonen T, Brodrick HJ, Harris SR, Corander J, Brown NM, Martin V, et al. Systematic longitudinal survey of invasive Escherichia coli in England demonstrates a stable population structure only transiently disturbed by the emergence of ST131. Genome Res. 2017;27(8):1437-49.

10. Nakamura A, Komatsu M, Ohno Y, Noguchi N, Kondo A, Hatano $\mathrm{N}$. Identification of specific protein amino acid substitutions of extended-spectrum $\beta$-lactamase (ESBL)-producing Escherichia coli ST131: a proteomics approach using mass spectrometry. Sci Rep. 2019;9(1):8555.

11. McNally A, Kallonen T, Connor C, Abudahab K, Aanensen DM, Horner $C$, et al. Diversification of colonization factors in a multidrug-resistant Escherichia coli lineage evolving under negative frequency-dependent selection. MBio. 2019;10(2):e00644-19.

12. Overdevest I, Haverkate M, Veenemans J, Hendriks Y, Verhulst C, Mulders A, et al. Prolonged colonisation with Escherichia coli O25:ST131 versus other extended-spectrum beta-lactamase-producing E. coli in a longterm care facility with high endemic level of rectal colonisation, the Netherlands, 2013 to 2014. Eurosurveillance. 2016;21(42):30376.

13. Kondratyeva K, Salmon-Divon M, Navon-Venezia S. Meta-analysis of pandemic Escherichia coli ST131 plasmidome proves restricted plasmid-clade associations. Sci Rep. 2020;10(1):36.

14. Merino I, Hernández-García M, Turrientes M-C, Pérez-Viso B, LópezFresneña N, Diaz-Agero C, et al. Emergence of ESBL-producing Escherichia coli ST131-C1-M27 clade colonizing patients in Europe. J Antimicrob Chemother. 2018;73(11):2973-80.

15. de Kraker MEA, Davey PG, Grundmann H, BURDEN study group. Mortality and hospital stay associated with resistant Staphylococcus aureus and Escherichia coli bacteremia: estimating the burden of antibiotic resistance in Europe. PLoS Med. 2011:8(10):e1001104.

16. Stewardson AJ, Allignol A, Beyersmann J, Graves N, Schumacher M, Meyer $R$, et al. The health and economic burden of bloodstream infections caused by antimicrobial-susceptible and non-susceptible Enterobacteriaceae and Staphylococcus aureus in European hospitals, 2010 and 2011: a multicentre retrospective cohort study. Euro Surveill. 2016;21(33):30319.

17. Tschudin-Sutter S, Lucet J-C, Mutters NT, Tacconelli E, Zahar JR, Harbarth S. Contact precautions for preventing nosocomial transmission of extended-spectrum $\beta$ lactamase-producing Escherichia coli: a point/ counterpoint review. Clin Infect Dis. 2017;65(2):342-7.

18. Schürch AC, Arredondo-Alonso S, Willems RJL, Goering RV. Whole genome sequencing options for bacterial strain typing and epidemiologic analysis based on single nucleotide polymorphism versus gene-bygene-based approaches. Clin Microbiol Infect. 2018;24(4):350-4.

19. The European Committee on Antimicrobial Susceptibility Testing. Breakpoint tables for interpretation of MICs and zone diameters. Version 7.1. http://www.eucast.org. Published 2017. Accessed 2021.

20. Lagacé-Wiens $P$, Tailor F, et al. Evaluation of a chromogenic medium for extended-spectrum beta-lactamase (ESBL)-producing Enterobacteriaceae. Clin Microbiol Infect. 2010;16(Suppl 2):S151-2.

21. François P, Bonetti E-J, Fankhauser C, Baud D, Cherkaoui A, Schrenzel J, et al. Rapid identification of ST131 Escherichia coli by a novel multiplex real-time allelic discrimination assay. J Microbiol Methods. 2017;140:12-4.

22. Johnson JR, Johnston BD, Porter SB, Clabots C, Bender TL, Thuras P, et al. Rapid emergence, subsidence, and molecular detection of Escherichia coli sequence type 1193-fimH64, a new disseminated multidrugresistant commensal and extraintestinal pathogen. J Clin Microbiol. 2019;57(5):e01664-18.

23. Epitools - Test chi-carré pour détecter une tendance. https:/epitools. ausvet.com.au/. Published 2018. Accessed 2021.

24. Dumyati G, Stone ND, Nace DA, Crnich CJ, Jump RLP. Challenges and strategies for prevention of multidrug-resistant organism transmission in nursing homes. Curr Infect Dis Rep. 2017;19(4):18.

25. Han JH, Goldstein EJC, Wise J, Bilker WB, Tolomeo P, Lautenbach E. Epidemiology of carbapenem-resistant Klebsiella pneumoniae in a network of long-term acute care hospitals. Clin Infect Dis. 2017;64(7):839-44.

26. Fisch J, Lansing B, Wang L, Symons K, Cherian K, McNamara S, et al. New acquisition of antibiotic-resistant organisms in skilled nursing facilities. J Clin Microbiol. 2012;50(5):1698-703.

27. March A, Aschbacher R, Dhanji H, Livermore DM, Böttcher A, Sleghel F, et al. Colonization of residents and staff of a long-term-care facility and adjacent acute-care hospital geriatric unit by multiresistant bacteria. Clin Microbiol Infect. 2010;16(7):934-44.

28. Andersson H, Lindholm C, Iversen A, Giske CG, Örtqvist Å, Kalin M, et al. Prevalence of antibiotic-resistant bacteria in residents of nursing homes in a Swedish municipality: healthcare staff knowledge of and adherence to principles of basic infection prevention. Scand J Infect Dis. 2012;44(9):641-9.

29. Pasricha J, Koessler T, Harbarth S, Schrenzel J, Camus V, Cohen G, et al. Carriage of extended-spectrum beta-lactamase-producing 
enterobacteriacae among internal medicine patients in Switzerland. Antimicrob Resist Infect Control. 2013;2:20.

30. Welker S, Boutin S, Miethke T, Heeg K, Nurjadi D. Emergence of carbapenem-resistant ST131 Escherichia coli carrying blaOXA-244 in Germany, 2019 to 2020. Eurosurv. 2020;25(46):2001815.

31. Decano AG, Downing T. An Escherichia coli ST131 pangenome atlas reveals population structure and evolution across 4,071 isolates. Sci Rep. 2019;9(1):17394

32. Colmenarejo C, Hernández-García M, Muñoz-Rodríquez JR, Huertas N, Navarro FJ, Mateo AB, et al. Prevalence and risks factors associated with ESBL-producing faecal carriage in a single long-term-care facility in Spain: emergence of CTX-M-24- and CTX-M-27-producing Escherichia coli ST131-H30R. J Antimicrob Chemother. 2020;75(9):2480-4.

33. Matsumura Y, Pitout JDD, Peirano G, DeVinney R, Noguchi T, Yamamoto $\mathrm{M}$, et al. Rapid identification of different Escherichia coli sequence type 131 clades. Antimicrob Agents Chemother. 2017;61 (8):e00179-e217.

34. Roer L, Hansen F, Thomsen MCF, Knudsen JD, Hansen DS, Wang M, et al. WGS-based surveillance of third-generation cephalosporin-resistant Escherichia coli from bloodstream infections in Denmark. J Antimicrob Chemother. 2017:72(7):1922-9.
35. Matsumura Y, Johnson JR, Yamamoto M, Nagao M, Tanaka M, Takakura S, et al. CTX-M-27- and CTX-M-14-producing, ciprofloxacin-resistant Escherichia coli of the $\mathrm{H} 30$ subclonal group within ST131 drive a Japanese regional ESBL epidemic. J Antimicrob Chemother. 2015;70(6):1639-49.

36. Peirano G, Lynch T, Pitout JDD, et al. Trends in population dynamics of Escherichia coli sequence type 131, Calgary, Alberta, Canada, 2006-2016. Emerg Infect Dis J. 2020;26(12):2907.

37. Terveer EM, Fallon M, Kraakman MEM, Ormond A, Fitzpatrick M, Caljouw MAA, et al. Spread of ESBL-producing Escherichia coli in nursing home residents in Ireland and the Netherlands may reflect infrastructural differences. J Hosp Infect. 2019;103(2):160-4.

38. Blom A, Ahl J, Månsson F, Resman F, Tham J. The prevalence of ESBLproducing Enterobacteriaceae in a nursing home setting compared with elderly living at home: a cross-sectional comparison. BMC Infect Dis. 2016;16(1):111.

\section{Publisher's Note}

Springer Nature remains neutral with regard to jurisdictional claims in published maps and institutional affiliations.
Ready to submit your research? Choose BMC and benefit from:

- fast, convenient online submission

- thorough peer review by experienced researchers in your field

- rapid publication on acceptance

- support for research data, including large and complex data types

- gold Open Access which fosters wider collaboration and increased citations

- maximum visibility for your research: over 100M website views per year

At BMC, research is always in progress.

Learn more biomedcentral.com/submissions 